\title{
Cytogenetic mechanism and genetic consequences of thelytoky in the wasp Trichogramma cacoeciae
}

\author{
F Vavre ${ }^{1}$, JH de Jong ${ }^{2}$ and R Stouthamer ${ }^{3}$ \\ ${ }^{1}$ Laboratoire de Biométrie et Biologie Evolutive, UMR-CNRS 5558, Université Claude Bernard Lyon I, 43 Boulevard du 11 Novembre \\ 1918, 69622 Villeurbanne Cedex, France; 'Laboratory of Genetics, Wageningen University, Arboretumlaan 4, 6703 BD Wageningen, \\ The Netherlands; ${ }^{3}$ Department of Entomology, University of California, Riverside, CA 92521, USA
}

In Hymenoptera, complete parthenogenesis, that is thelyto$\mathrm{ky}$, is a common phenomenon where virgin females produce only daughters. Thelytoky is often induced by bacteria of the genus Wolbachia, but can also be genetically determined by the insect itself, as in the genus Trichogramma where both forms exist. In order to compare these two forms of thelytoky, chromosome behaviour analysis in young eggs and genetic analysis of microsatellite markers were carried out in the wasp Trichogramma cacoeciae, where thelytoky is genetically determined. Microscopic studies revealed that during female gamete formation meiotic cells undergo only a single equational division followed by the expulsion of a single polar body. This absence of meiotic recombination and reduction corresponds well with the high levels of heterozygosity observed in females collected from the field and a nonsegregation pattern in the offspring of heterozygous females. We therefore concluded that diploidy in T. cacoeciae is maintained through an apomictic cloning mechanism and that the incidence of thelytoky under genetic control of the wasp differs entirely from the mechanism induced by Wolbachia infection, where thelytoky is restored through gamete duplication.

Heredity (2004) 93, 592-596. doi:10.1038/sj.hdy.6800565

Published online 25 August 2004

Keywords: parthenogenesis; apomixis; cytogenetics; Trichogramma; genetic diversity; Wolbachia

\section{Introduction}

The most common form of reproduction in the insect order Hymenoptera is arrhenotoky, where haploid males develop from unfertilised eggs and diploid females develop from fertilised eggs. However, numerous species exhibit thelytoky, a form of complete parthenogenesis where unfertilised eggs develop into diploid females (Luck et al, 1992). Suomalainen et al (1987) and Gottlieb et al (2002) described different mechanisms accounting for such parthenogenetic development, but differing in their consequences for genetic diversity: (1) Apomixis resembles a normal mitosis with only a single maturation division occurring in the oocyte. Recombination is generally absent, resulting in the conservation of heterozygosity in the offspring and clonal reproduction of females. (2) Automixis, where meiotic reduction is compensated, produces diploid gametes by premeitotic doubling, fusion of two of the four meiotic nuclei, or fusion of cleaved nuclei and results in decline of heterozygosity in the offspring, except for the case of premeiotic doubling where offspring is genetically identical to the mother. While apomixis has been observed in several orders of insects, it has only been observed in a few species in Hymenoptera where

Correspondence: F Vavre, Laboratoire de Biométrie et Biologie Evolutive, UMR CNRS 5558, Université Claude Bernard Lyon I, 43 Boulevard du 11 Novembre 1918, 69622 Villeurbanne Cedex, France.

E-mail:vavre@biomserv.univ-lyon1.fr

Received 30 April 2003; accepted 23 June 2004; published online 25 August 2004 automixis seems to prevail (Suomalainen et al, 1987; Beukeboom and Pijnacker, 2000).

Thelytoky can be under the control of the insect itself or its endosymbionts (Stouthamer et al, 1990a, 1997). Among the different microorganisms that have been identified (Stouthamer et al, 1993; Weeks et al, 2001; Zchori-Fein et al, 2001), bacteria of the genus Wolbachia appear to be the most common parthenogenesis inducers. This symbiotic form of thelytoky can easily be distinguished from the thelytoky under direct control of the insect by its revertibility. In microbe-induced parthenogenesis, elimination of the symbiont through antibiotic or heat treatment allows females to produce males, whereas in the nonrevertible thelytoky, induction of male production is not possible (Stouthamer et al, 1990b).

In the tiny wasps of the genus Trichogramma (Hymenoptera: Trichogrammatidae), both forms of thelytoky are known (Stouthamer et al, 1990b). It is not known whether they are related, but one can imagine that nonrevertible parthenogenesis could have arisen from the integration of one (or more) bacterial gene(s) in the insect genome. This scenario has already been proposed in Isopods where both Wolbachia infection and nuclear factors, potentially acquired from Wolbachia, can cause feminisation (review in Rigaud, 1997). Recently, Kondo et al (2002) found evidence for transfer of Wolbachia genes into the host genome.

One possible way to explore if the two forms of thelytoky are related is to compare their cytogenetic mechanisms. In Trichogramma, restoration of diploidy in Wolbachia-induced thelytoky arises from the nonsegregation of chromosomes 
during the first mitotic division of the development by fusion of cleaved nuclei and consequently produced totally homozygous females (Stouthamer and Kazmer, 1994)

Here, we describe the cytogenetic mechanism of thelytoky in Trichogramma cacoeciae and the consequences for its genetic diversity. The species exhibits complete parthenogenesis and males are absent in natural populations. Pinto (1998) observed only five males out of approximately 15000 individuals under laboratory conditions. Males cannot be induced by antibiotic or heat treatment, and neither Wolbachia nor other symbionts have ever been found (Stouthamer et al, 1990b). Our results show that, in $T$. cacoeciae, the cytogenetic mechanism of thelytoky and the consequences of asexuality for genetic diversity and heterozygosity clearly differ from what is observed in Wolbachia-induced thelytoky, suggesting an independent evolutionary origin of the two forms of thelytoky.

\section{Material and methods}

\section{Strain and rearing}

One line of $T$. cacoeciae (Y190) was established from a single female originating from a field-collected host in Wageningen (The Netherlands) in 1994. Wasp culture was maintained on irradiated Ephestia kuehniella eggs (supplied by Koppert bv, Berkel \& Rodenrijs, The Netherlands).

\section{Cytogenetic techniques}

Female wasps were fed honey for 1 day after emergence. Several hundreds of females were given an egg sheet containing 10 Mamestra brassicae eggs. After 30-45 min, the egg sheet was removed and wasps remaining on the egg sheet were brushed-off. The egg sheet was kept for a variable time span (from 0 to $4 \mathrm{~h}$ ) to allow the Trichogramma eggs to develop. Host eggs were opened in a drop of water on a microscope slide allowing the recovery of up to 30 wasp eggs. Host debris was then removed, and wasp eggs were dechorionated in bleachwater (1:250 commercial bleach solution:water) for $3 \mathrm{~min}$, fixed in $60 \%$ glacial acetic acid for $3 \mathrm{~min}$ and finally covered with freshly prepared Carnoy's fixative (3:1 of absolute ethanol:glacial acetic acid). After air drying, slides were carefully rinsed with $70 \%$ ethanol and air dried again. DNA was then stained with DAPI (100 ng/ $\mathrm{ml}$ ) in Vectashield ${ }^{\mathbb{R}}$ (Vector Laboratories, Burlingame, CA, USA). Slides were covered with a coverslip and studied under an epifluorescence microscope with DAPI filter and $\times 100$ Plan Apo objective. Selected images were photographed on 400 ISO negative film and scanned for digital processing

\section{Genetic markers and PCR reaction}

Four sets of microsatellite markers were used (Table 1), either to study segregation in the offspring of isolated females or to study the genetic diversity in the field (see below). For DNA extraction, one individual was ground in $50 \mu \mathrm{l}$ of $5 \%$ Chelex solution. Samples were kept for $6 \mathrm{~h}$ at $56^{\circ} \mathrm{C}$. After $10 \mathrm{~min}$ at $95^{\circ} \mathrm{C}$, samples were centrifuged and $2-4 \mu \mathrm{l}$ of the supernatant were used for PCR reactions in $25 \mu \mathrm{l}$ final volume containing $200 \mathrm{mM}$ dNTP, $10 \mathrm{pM}$ primers (forward primer labelled with $\mathrm{Cy} 3$ fluorochrome), 1 IU taq DNA polymerase, and 2-4 $\mu \mathrm{l}$ of template DNA. PCR conditions were $5 \mathrm{~min}$ at $95^{\circ} \mathrm{C}$, then 35 cycles: $1 \mathrm{~min}$ at $95^{\circ} \mathrm{C}, 1 \mathrm{~min}$ at annealing temperature (Table 1) and $1 \mathrm{~min}$ at $72^{\circ} \mathrm{C}$. PCR reaction was ended by a 10 min elongation at $72{ }^{\circ} \mathrm{C}$. The amplified DNA samples were analysed on a polyacrilamide gel using an automatic sequencer and GeneScan software (Applied Biosystems).

\section{Segregation experiments}

In total, 15 females from the line Y190 were isolated and allowed to oviposit on E. kuehniella eggs for 5 days. DNA from mothers and daughters was then extracted, and all individuals were genotyped using the TAC47 microsatellite primers.

\section{Genetic diversity in the field}

Collection in the field was performed around Wageningen, The Netherlands using Mamestra brassicae eggs glued on cards (van Rijswijk et al, 2000). Despite the absence of males, identification of T. cacoeciae is straightforward using the sequence of the internal transcribed spacer 2 (Pinto et al, 2002). The emerged T. cacoeciae individuals were females, which were used to initiate isofemale lines. Since one egg card could have been parasitised by one or several females, we only studied a single line per egg-card. In the end, we could analyse the genotype of 14 field females. Despite the low number of individuals, we performed a parsimony analysis using PAUP 4.0b2a (Swofford, 2001) to reconstruct the history of these 14 genotypes.

\section{Results}

\section{Cytogenetic analysis}

Each parasitized M. brassicae egg contains about $30 \mathrm{~T}$. cacoeciae embryos. This allowed us to look at several hundreds of embryos and consequently each stage of

Table 1 Microsatellite loci studied and genetic diversity measured on fourteen field-collected females

\begin{tabular}{|c|c|c|c|c|}
\hline & TTG 46 & TAC 47 & TTC 53 & CT 122 \\
\hline \multirow[t]{2}{*}{ Forward primer } & 5'-GATGTTTACT & 5'-GGGCATGTGC & 5'-GTGACTGCCT & $5^{\prime}$-CTACGGCGAC \\
\hline & TCGCAGGCCGC & AAAAGACTAG & TATTCTGCATAC & AATTGCCAC \\
\hline \multirow[t]{2}{*}{ Reverse primer } & 5'-CTACGGGGCA & 5'-CGAGAGGGTTT & 5'-TCGGGTCGTT & 5'-CATCTTGGTC \\
\hline & TACGATATGTG & CTTCAATGG & GTAGCGGGC & GAACCGAGCAG \\
\hline Annealing temp. $\left({ }^{\circ} \mathrm{C}\right)$ & 66 & 58 & 58 & 66 \\
\hline Number of alleles & 5 & 10 & 4 & 2 \\
\hline $\min -\max$ freq. & $0.14-0.29$ & $0.04-0.25$ & $0.05-0.65$ & $0.07-0.93$ \\
\hline Heterozygosity & 0.5 & 0.93 & 0.4 & 0.14 \\
\hline
\end{tabular}

Only the frequencies of the least and the most common alleles are given for each locus. 

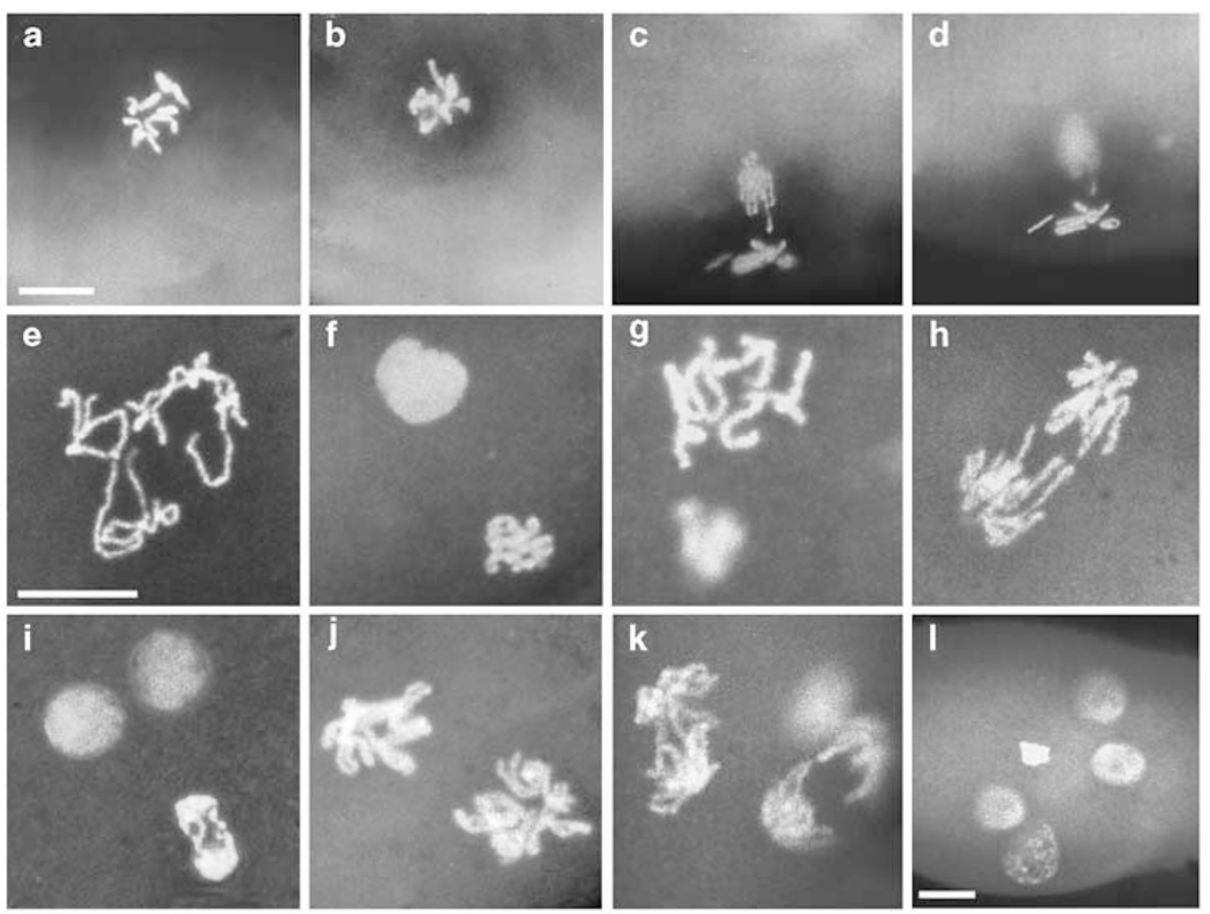

Figure 1 Meiosis and first mitotic divisions in Trichogramma cacoeciae. Bars indicates $10 \mu \mathrm{m}$. (a-d) same scale, (e-k) same scale. (a and b) Compact first metaphase. (c and d) First anaphase. (e) Central nucleus after the first anaphase showing 10 chromosomes. (f) Central nucleus in interphase indicating the end of meiosis and a single polar body. (g) Central nucleus in metaphase. (h) Central nucleus in anaphase of the first mitotic division. (i) Two central nuclei in interphase and a single polar body showing partial division. (j) Two central nuclei in metaphase of the second mitotic division. (k) Anaphase of the second mitotic division. (l) Four nuclei in interphase and a single polar body.

development has been observed several times. Early embryonic development of Trichogramma eggs have recently been characterized in T. kaykai (Van Vugt et al, 2003). We observed roughly the same time scale for development in T. cacoeciae. In newly laid eggs, we observed condensed chromosomes at meiotic prophase I (Figure 1a). The clumped chromosome mass did not allow us to study chromosome pairing. In approximately 30-min-old eggs, the nuclei decondense and orient towards the equatorial plate (Figure 1b). Although individual chromosomes are difficult to assess, we counted about 10 chromosomes in the complement, a diploid chromosome number also known from other Trichogramma species (Stouthamer and Kazmer, 1994; Van Vugt et al, 2003). Therefore, there is no doubling of the chromosome number before meiosis. Anaphase cells were rarely observed, which suggest that the stage lasts a very short time. One set of chromosomes lies close to the periphery of the egg and forms the polar body I, while the other set, with about 10 discernable chromosomes, lies in the central part (Figure 1c-d). We never observed cell complements with the haploid chromosome number $(n=5)$ indicating that meiosis is exclusively nonreductional (Figure 1e). After a short lasting interphase the central nucleus went through a mitotic division with 10 doubled chromosomes at metaphase (Figure 1f) and two sets of chromatids at anaphase (Figure $1 \mathrm{~g}-\mathrm{h}$ ), indicating that meiosis is replaced by a single equational division followed by the expulsion of a single polar body. None of the two nuclei that entered interphase (Figure 1i) resulted in the polar body II, instead they divided again mitotically giving rise to four nuclei (Figure $1 \mathrm{j}-1$ ). At the same time, the chromosomes of the polar body I separated without displaying a clear mitosis.

\section{Genetic analysis}

Genetic analyses of females from line Y190 revealed heterozygosity for the TAC47 locus in all 15 mothers and 64 daughters tested. This suggests suppression of recombination of the locus due to its position in the achiasmatic centromere region or by the total absence of meiotic recombination during the 2 years this isofemale line was maintained in the laboratory.

Tests on the genetic variability and heterozygosity of the four microsatellite loci in 14 field-collected females are presented in Table 1. Number of alleles per locus varies from 2 to 10, and heterozygosity ranged from 0.14 for CT122 to 0.93 for TAC47. Moreover, in the 14 females that were tested we detected 11 different profiles, showing that populations are composed of several different genotypes, and that genetic diversity in these markers is relatively high. To gain insight into the history of these fourteen genotypes, we used parsimony to analyse their relationships. We obtained 12 equally parsimonious trees. These trees have a length of 22, while the minimum length is 20 , indicating that only two homoplasies are necessary to reconstruct the phylogenetic relationships among the 14 females. Even though based on only 14 individuals, this analysis suggests that recombination does not take place, that sex is either absent or extremely rare, and that asexuality probably arose from a single event in this species. 


\section{Discussion}

Our results strongly suggest achiasmatic meiosis in the thelytokous T. cacoeciae because: (i) meiosis is achiasmatic with only a single equational division and expulsion of a single polar body; (ii) heterozygous females do not segregate for microsatellite markers in their offspring; (iii) field-collected females display moderate to high levels of heterozygosity. This mechanism is thus typically apomictic and differs substantially from Wolbachiainduced parthenogenesis insect systems where diploidy is restored through an automixis resulting in homozygous females in a single generation (Stille and Dävring, 1980; Stouthamer and Kazmer, 1994; Gottlieb et al, 2002) and therefore suggests that Wolbachia and genetically determined thelytoky in Trichogramma do not have a common evolutionary origin.

Thelytoky in T. cacoeciae could have originated from interspecific hybridisation (Pinto and Stouthamer, 1994), which has been described for $T$. vogelei $\times T$. brassicae (Pintureau and Babault, 1981) and T. exiguum $\times T$. californicum (Nagarkatti, 1970). Hybridisation-induced parthenogenesis is likely caused by epistatic interactions between parental genomes. Lack of recombination (as observed in $T$. cacoeciae) between the parental genomes can make epistatic interactions fixed and could allow the maintenance of thelytoky.

The great genetic diversity, based on the large number of different genotypes in this small population sample, can be explained by different factors. Firstly, heterozygosity resulting from incidental sexual reproduction may be maintained through generations in the absence of recombination, as suggested in thelytokous species of the genus Lysiphlebus (Belshaw et al, 1999; Belshaw and Quicke, 2003). However, recombination has not been detected among the 14 females tested, and moreover apomixis should lead in an increase of ploidy if rare sex occurs (Belshaw and Quicke, 2003), which has not been observed. Secondly, mutation-drift equilibrium could be in favour of mutations, which implies that the effective size of the population is sufficiently high to maintain an important allelic diversity (Kimura, 1983). It would be very interesting to have a clearer picture of the genetic diversity in $T$. cacoeciae at a larger geographical scale. Comparison between mitochondrial and nuclear markers could help in resolving this issue, since the same patterns are expected for the two compartments only if rare sex does not occur.

In any case, the mechanism that maintains diploidy prevents the formation of homozygotes and thus the expression of recessive lethal mutations, reducing the negative impact of the lack of sexual reproduction. To what extent this may explain why T. cacoeciae shows such a wide geographic distribution remains to be studied (Pintureau, 1997). However, since the genotypes are fixed in the course of generations (no recombination occurs), there is little space for the creation of new genetic combinations and T. cacoeciae can be expected to respond to selection slower than sexual taxa, unless rare sex occurs and then creates new combinations.

\section{Acknowledgements}

We thank the European Union (ERBIC Project FAIR5CT97-3489 and Euwol project QLK3CT-2000-01079) for financial support, Manolo Gouy for helpful advices on the genetic analysis and Catherine Lœvenbruck for her help in the processing of images.

\section{References}

Belshaw R, Quicke DLJ (2003). The cytogenetics of thelytoky in a predominantly asexual parasitoid with covert sex. Genome 46: 170-173.

Belshaw R, Quicke DLJ, Völk PW, Godfray HCJ (1999). Molecular markers indicate rare sex in a predominantly asexual parasitoid wasp. Evolution 53: 1189-1199.

Beukeboom LW, Pijnacker LP (2000). Automictic parthenogenesis in the parasitoid Venturia canescens (Hymenoptera: Ichneumonidae) revisited. Genome 43: 939-944.

Gottlieb Y, Zchori-Fein E, Werren JH, Karr TL (2002). Diploidy restoration in Wolbachia-infected Muscidifurax uniraptor (Hymenoptera: Pteromalidae). J Invert Path 81: 166-174.

Kimura M (1983). The Neutral Theory of Molecular Evolution, Cambridge University Press: Cambridge.

Kondo N, Nikoh N, Ijichi N, Shimada M, Fukatsu T (2002). Genome fragment of Wolbachia endosymbiont transferred to X chromosome of host insect. Proc Natl Acad Sci USA 99: 14280-14285.

Luck RF, Stouthamer R, Nunney L (1992). Sex determination and sex ratio patterns in parasitic hymenoptera. In: Wrench DL, Ebbert MA (eds) Evolution and Diversity of Sex Ratio in Haplodiploid Insects and Mites, Chapman \& Hall: New York. pp 442-476.

Nagarkatti S (1970). The production of a thelytokous hybrid in an interspecific cross between two species of Trichogramma. Current Science 39: 76-78.

Pinto JD (1998). Systematics of the North American species of Trichogramma (Hymenoptera: Trichogrammatidae). Memoirs of the Entomological Society of Washington, Allen Press Inc.: Washington, DC. No. 22, 287pp.

Pinto JD, Koopmanschap AB, Platner GR, Stouthamer R (2002). The North American Trichogramma (Hymenoptera: Trichogrammatidae) parasitizing certain Tortricidae (Lepidoptera) on apple and pear, with ITS2 DNA characterizations and description of a new species. Biol Control 23: 134-142.

Pinto JD, Stouthamer R (1994). Systematics of the Trichogrammatidae with emphasis on Trichogramma. In: Wajnberg E, Hassan SA (eds) Biological Control with Egg Parasitoids, CAB International: Ascot. pp 1-36.

Pintureau B (1997). Systematic and genetical problems revised in two closely related species of Trichogramma, T. embryophagum and T. cacoeciae (Hym., Trichogrammatidae). Miscellania Zoologica 20: 11-18.

Pintureau B, Babault M (1981). Caractérisation enzymatique de Trichogramma evanescens et T. maidis, étude des hybrides. Entomophaga 26: 11-22.

Rigaud T (1997). Inherited microorganisms and sex determination of arthropod hosts. In: O'Neill SL, Werren JH, Hoffmann AA (eds) Influential passengers, Oxford University Press: New York. pp 81-101.

Stille B, Dävring L (1980). Meiosis and reproductive strategy in the parthenogenetic gall wasp Diplolepis rosae (Hymenoptera, Cynipidae). Hereditas 92: 353-362.

Stouthamer R (1997). Wolbachia-induced parthenogenesis. In: $\mathrm{O}^{\prime}$ Neill SL, Werren JH, Hoffmann AA (eds) Influential Passengers, Oxford University Press: New York. pp 102-124.

Stouthamer R, Breeuwer JAJ, Luck RF, Werren JH (1993). Molecular identification of microorganisms associated with parthenogenesis. Nature 361: 66-68.

Stouthamer R, Kazmer DJ (1994). Cytogenetic of microbe associated parthenogenesis, consequences for gene flow in Trichogramma wasps. Heredity 73: 317-327.

Stouthamer R, Luck RF, Hamilton WD (1990a). Antibiotics cause parthenogenetic Trichogramma to revert to sex. Proc Natl Acad Sci USA 87: 2424-2427. 
Stouthamer R, Pinto JD, Platner GR, Luck RF (1990b). Taxonomic status of thelytokous forms of Trichogramma (Hymenoptera: Trichogrammatidae). Annals of the Entomological Society of America 83: 475-481.

Suomalainen E, Saura A, Lokki J (1987). Cytology and Evolution in Parthenogenesis, CRC Press: Boca Raton.

Swofford DL (2001). PAUP. Phylogenetic Analysis Using Parsimony Version 4. Sinauer Associates: Sunderland, Massachusetts.

Van Rijswijk M, van der Heijden R, Jongema Y, Stouthamer R (2000). Occurrence and molecular identification of the Dutch Trichogramma species. Proc Exper \& Appl Entomol, NEV Amsterdam 11: 67-70.
Van Vugt JFA, Salverda M, De Jong JH, Stouthamer R (2003). The paternl sex ratio chromosome in the parasitic wasp Trichogramma kaykai condenses the paternal chromosomes into a dense chromatin mass. Genome 46: 580-587.

Weeks AR, Marec F, Breeuwer JAJ (2001). A mite species that consists entirely of haploid females. Science 292: 2479-2482.

Zchori-Fein E, Gottlieb Y, Kelly SE, Brown JK, Wilson JM, Karr TL, Hunter MS (2001). A newly discovered bacterium associated with parthenogenesis and a change in host selection behavior in parasitoid wasps. Proc Natl Acad Sci USA 98: 12555-12560. 